\title{
A low-cost smart egg-incubator
}

\author{
Benjamin Kommey ${ }^{1 *}$, Daniel Akudbilla ${ }^{1}$, Godfred Doe $^{\mathbf{1}}$, Clifford Owusu Amponsah $^{\mathbf{1}}$ \\ ${ }^{1}$ Department of Computer Engineering, Kwame Nkrumah University of Science and Technology, Kumasi, Ghana
}

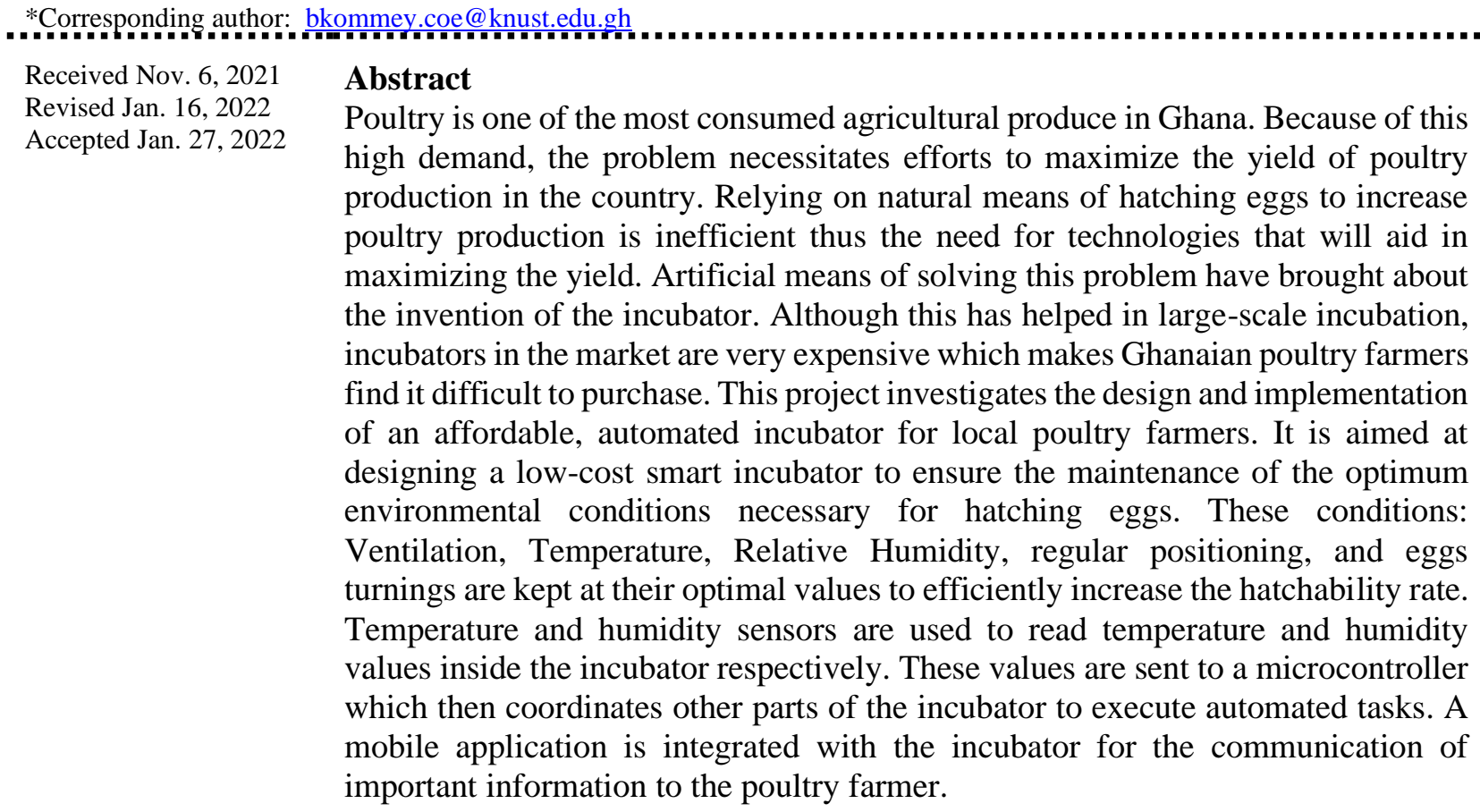

(C) The Author 2022.

Keywords: Hatchability rate; Incubator; Temperature; Humidity; Relative

Published by ARDA.

humidity; Ventilation

\section{Introduction}

In Ghana, the demand for poultry meat is about $400.000 \mathrm{mt}$ and the local production is just about $57.871 \mathrm{mt}$. The country's poultry meat import is currently about $180.000 \mathrm{mt}$ (over USD 300 million annually) leaving a national demand shortfall of $162.129 \mathrm{mt}$. [1]. In the 1960s, the Government of Ghana (GoG) undertook an initiative to promote commercial poultry production. This resulted in the industry supplying about $95 \%$ of poultry meat and eggs in the country. Due to irregular supply of day-old chicks and outbreak of poultry diseases, growth was initially slow and broiler production has experienced a steep decline from $80 \%$ in 2000 to $10 \%$ in 2010 according to GoG sources [2]. Considering the high poultry meat import bill and the continuous decline in poultry production locally, the necessity arises to develop competitive and efficient means that will revitalize poultry farming and increase local production to reduce import bills and to contribute to employment creation. Broiler meat production has been identified as the fastest way to reduce meat imports. Rearing for Food and Jobs (RFJ) was initiated by the GoG in 2019 purposely for self-sufficiency in meat production. About GHC500 million (USD 87 million) has been invested to support broiler production revitalization according to Ghana's Agricultural Development Bank [3].

Artificial incubation technologies must come into play if this broiler production revitalization exercise is to yield effective results. Artificial incubators are used to hatch a larger number of eggs at a time. This increases

This work is licensed under a Creative Commons Attribution License (https://creativecommons.org/licenses/by/4.0/) that allows others to share and adapt the material for any purpose (even commercially), in any medium with an acknowledgement of the work's authorship and initial publication in this journal. 
the supply of day-old chicks, but these incubators are not widely used in Ghana. They are used by a few major poultry organizations. The available incubators are mostly too expensive for the local farmers.

This paper focuses on the design of a low-cost automated egg incubator (digiIncubator) which is highly efficient with a high hatchability. The project aims to maintain temperature and relative humidity at optimum values within the incubator chamber to achieve a higher hatchability rate. The relationship between heat and temperature is used to model the system. The system is well ventilated, while proper and periodic turning of eggs on all levels in the incubator chamber is ensured. Electricity is used to provide heat in the incubator by mean of electric bulbs.

\section{Related works}

In artificial incubation an Egg Incubator is used to provide the conditions that the brooder hen in nature gives to the eggs it broods on. These conditions need to be reproduced to nearly the same levels for the fertile eggs in the incubator to develop and hatch $[4,5]$. According to [6] and [7], conditions that need to be controlled to ensure proper incubation of a fertile egg are temperature, humidity, turning of eggs and ventilation.

The idea of artificial incubation has always been practiced by human beings since the time of the Egyptian empire and Chinese dynasties around 200BC [8]. During this time, the type of incubation used was manual which meant it was totally depended on human beings. Temperature changes were affected in the incubator by moving the eggs, adding additional eggs to use the heat of embryological development of older eggs, and by regulating the flow of fresh air through the hatching area. Shittue, Muhammad and Jimoh [9] used dht11 sensor in reading the temperature and humidity of the ambient. The dht11 is known to be an inaccurate temperature and humidity sensor as it is incapable of sensing fractional changes in temperature, thus for a system like the incubator which needs accuracy in temperature and humidity reading this sensor is not suitable [10]. Despite this, they had good ventilation system. The system has a mechanical and an electronic part. The mechanical part angularly tilts the egg trays up and down alternatively and hourly, using a de motor and limit switch sensor for angular movement control. The electronic part comprised of the microcontroller, sensors, and LCD display. Data from the sensors were processed by the microcontroller and the various control elements were activated to moderate the conditions in the incubator

[11] used image recognition to detect the fertility of eggs. Cameras used in capturing images increased the cost of the incubator. The incubation process was autonomous with respect to humidity and temperature control. An Arduino microcontroller interfaced and coded with LabView software was used. [12] worked on designing a smart incubator in which they used a nodeMCU as the wireless communication module for logging the necessary data to the cloud. It was realized that incorporating an incandescent lamp as the heat source reduced substantial power consumption as against using an electric heater. High efficiency fans were used to circulate the heated air within the chamber to maintain even temperature. A humidifier was used to maintain the pre-set humidity in the chambers of the incubator structure. A standard, widely available 16x2 LCD display unit was integrated into the electronic module that was used as a display unit. The outputs from all the sensors were connected to the heart of the incubator, which gave the command to power on or off the desired peripheral connected to it, as deemed fit. A redundant battery was also connected as a power backup during electrical power failures. IoT and nodeMCU were used to integrate on the smart phone that provided the overall monitoring and configuration to the user.

\section{Materials and methods}

\subsection{Digiincubator system architecture}

As shown in figure 1, the system architecture has three main parts: Smart Incubator (SI), the server (SVR), and the mobile software application (digiINCU).

The SI is the whole incubator with sensors, microcontroller, and wireless communication modules. These components are grouped as a unit, making the embedded hardware. The sensors are responsible for collecting the system essentials, temperature, and relative humidity of the incubator chambers. These sensors read the temperature and relative humidity values and send the values to the microcontroller. The data received from the sensors is sent to the server via the wireless communication medium to be displayed to the user on the mobile software application (digiINCU).

The microcontroller sends processed data to LCD for display. With this data, the microcontroller sets other working limits within which the incubator works optimally. The SVR is the point of communication between 
the incubator and the digiINCU. The server acts as the data storage and exchange point between the hardware and mobile application. The digiINCU retrieves data sent from the incubator to server and makes same available to the user. Via the digiINCU a user can remotely control the activities of the SI when deemed necessary. The data is then sent to the microcontroller to use in regulating the system parameters.
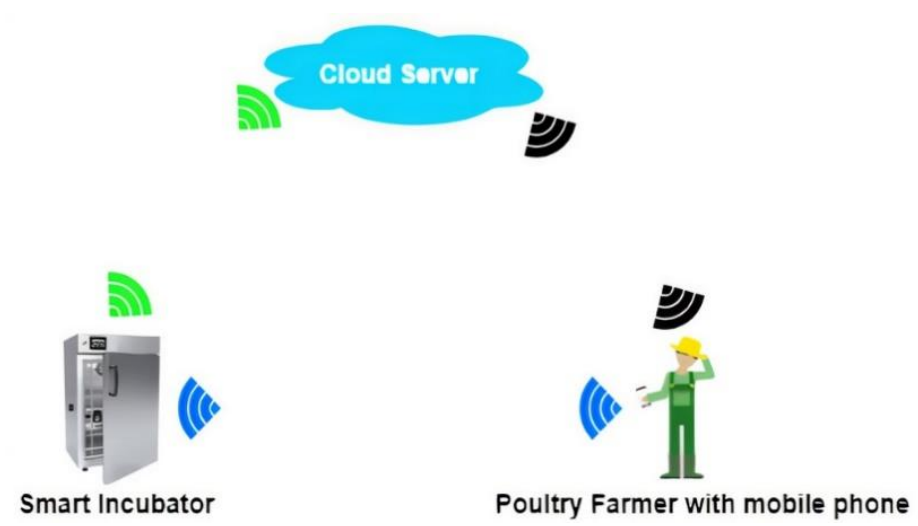

Figure 1. DigiIncubator system architecture

\subsection{DigiIncubator hardware block diagram}

The digiIncubator hardware block diagram illustrates the interconnection of the various hardware components that function together to accomplish the incubation process. At the start of the system by pressing the power button, the power from the source is regulated by 12 volts AC/DC converter to prevent excessive power supply to the microcontroller. The LED comes on to indicate that the system is powered up and ready. To select the type of egg to incubate, the user of the hardware uses the keypad to input the desired selection. The preset optimal environmental parameters associated with the selected egg type are then set. The DHT22 sensor, used to read the humidity and temperature of the atmospheric conditions of the incubator's ambient, reads these values and sends the data to the microcontroller. The microcontroller use the received data to perform logical operations based on which it regulates the relays connected to the electric bulbs, electric fans and the electric motor. The electric bulb is turned on when the temperature within the incubator chambers is below the optimum temperature for the particular egg being incubated and is turned off in the reversed situation based on the signal from the microcontroller to the connected relay. The electric motor is turned on and off periodically to supply the torque required by the turning mechanism to turn the eggs. To ensure that the air within the incubator is evenly spread and the relative humidity uniform, electric fans are installed in the incubator. The data collected by the sensors together with other operating data and alerts, are sent by the microcontroller via a wireless module to the mobile application of the user's smart phone. The data is also displayed on the LCD to a user near the incubator system setup. Fig. 2 depicts graphically, the block diagram of the incubator system.

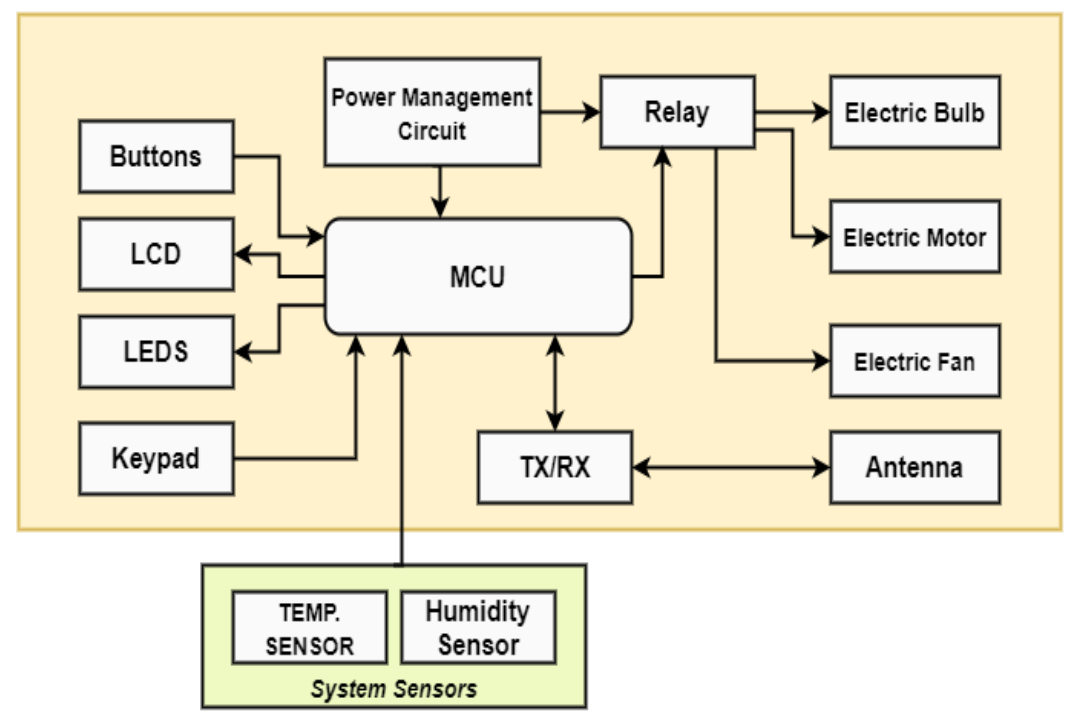

Figure 2. Digilncubator hardware block diagram 


\subsection{DigiIncubator system workflow}

The system workflow as shown in Fig. 3 is the sequential stages that the incubator system goes through to accomplish its desired purpose of hatching eggs. When the system is turned on the type of egg to be incubated is selected by the user via the mobile application or by using the keypad on the digiIncubator, the necessary parameters associated with the selected egg are then sent to the microcontroller and the incubation begins. Two workflows, the Sensor Workflow (SWF) and the Timing Workflow (TWF) are then initiated simultaneously.

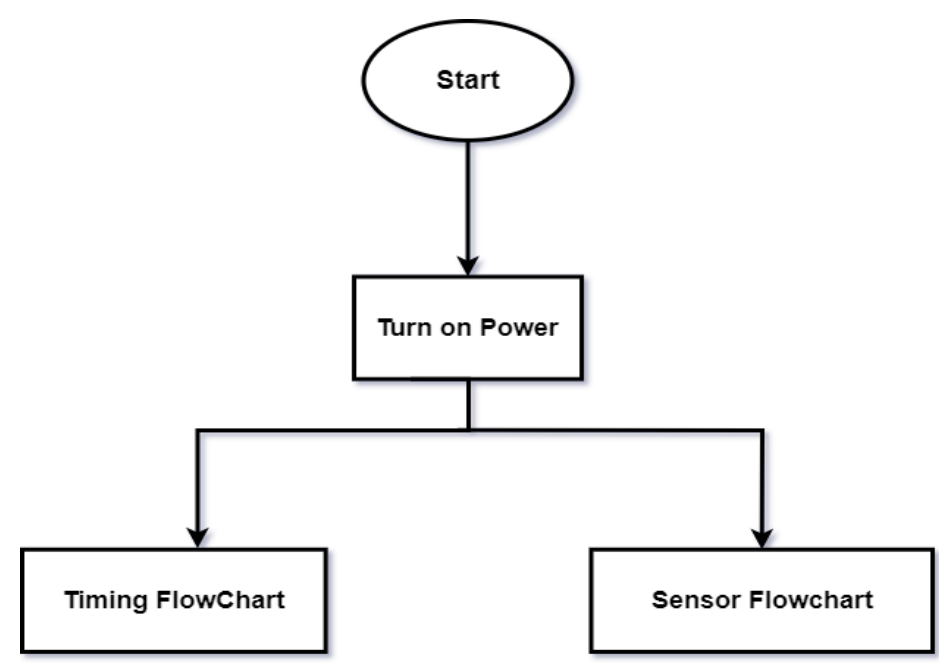

Figure 3. DigiIncubator system flowchart

\subsection{Sensor workflow (SWF)}

As shown in Fig. 4, the temperature and relative humidity data read from the incubator's ambient is compared with the optimum values associated with the particular type of egg being incubated. For instance, the optimal values for chicken eggs are $37.8^{\circ} \mathrm{C}$ and $60 \%$ relative humidity. When the temperature read by the sensor is lower than the optimum temperature value, the electric bulbs are turned on to supply heat to increase the temperature within the incubator otherwise the electric bulbs are turned off to prevent further heating and to lower the temperature. Likewise, a check is performed to determine whether the read relative humidity is less than the optimum relative humidity. When the read relative humidity is less, an alert is sent to the user to request the addition of water to the water-bowl. The collected ambient data is sent to the mobile application on the user's smart phone.

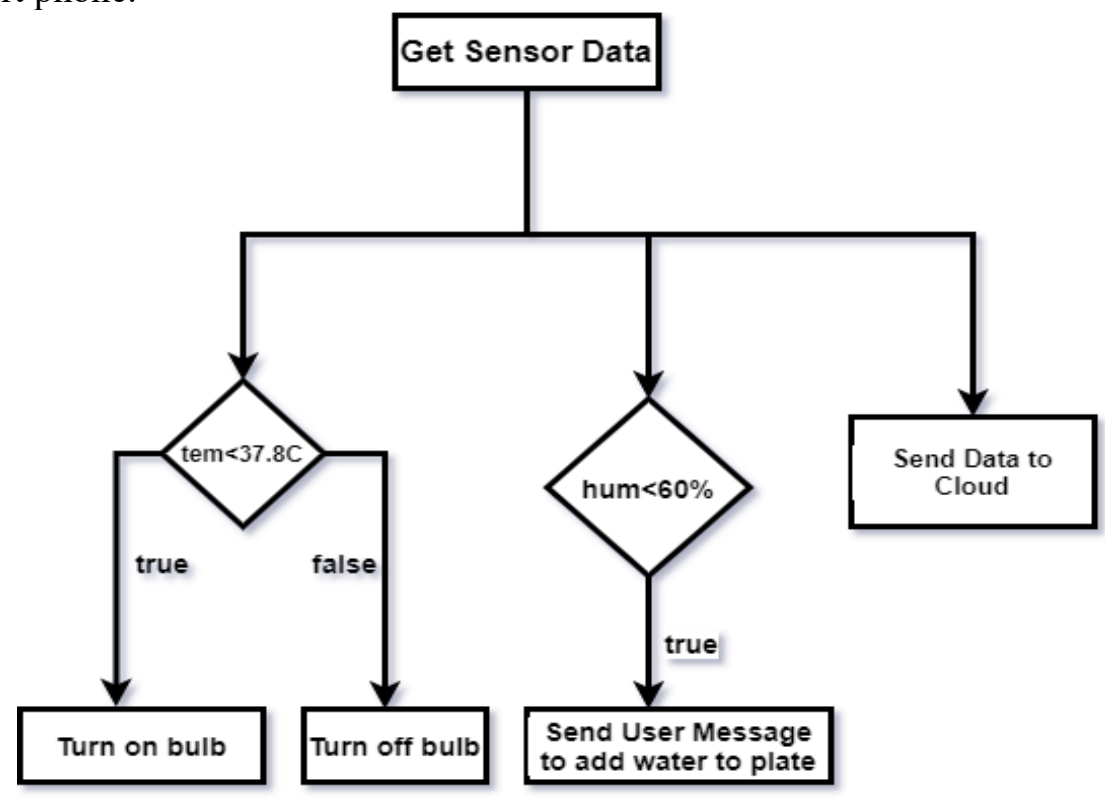

Figure 4. Sensor work flow (SWF)

In the timing workflow (TWF), as shown in Fig. 5, the number of days required to incubate the selected type of egg is sent to the microcontroller when the egg is selected. The electric fan is turned on and a timer to 
regulate the periodic turning of the eggs is set. A check is performed to determine if the time equals turn-time. If this condition is true, the eggs are turn and the time is reset. A check is also performed to determine if the number of days of incubation equals the number of days required for the incubation process. If this condition is true, the fan is turned on and the incubation is halted otherwise, the system continues operation and the checks are repeated.

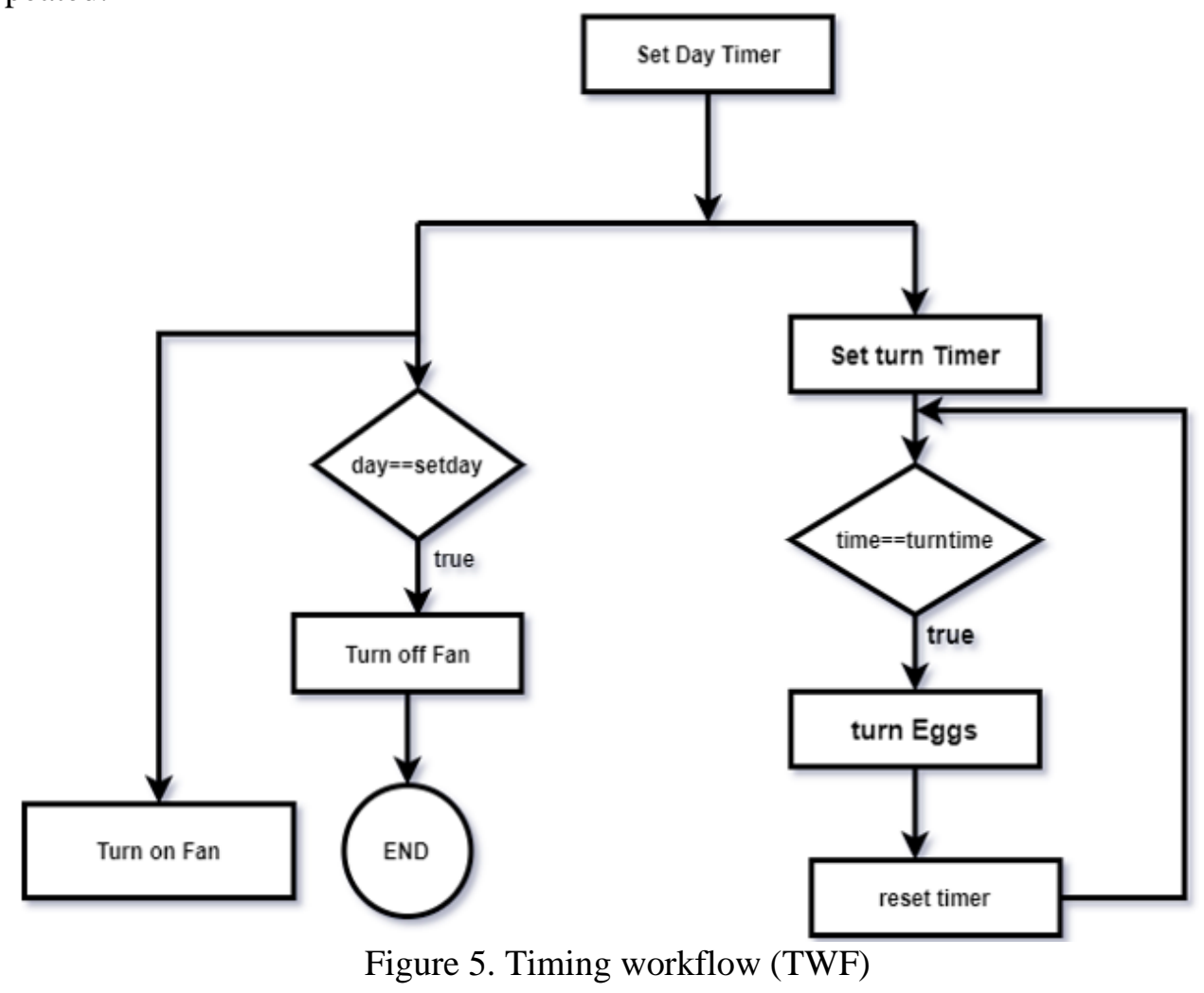

\subsection{DigiIncubator system design}

The digiIncubator system is modularly designed. Each part is separately designed and the individual parts are later put together to form the complete incubator system. The individual parts of the incubator ie. Tray support, Door panel, Back panel, Side panel, Top and Bottom panels and their functions are as described in table 1. The digiIncubator system was designed with wood and this helped achieve low heat loss thus saving energy, ensuring proper ventilation, and making it low cost.

\begin{tabular}{|c|l|}
\hline Module \\
Tray \\
support
\end{tabular}




\begin{tabular}{|l|l|}
\hline Module \\
\hline $\begin{array}{l}\text { Back } \\
\text { Panel }\end{array}$
\end{tabular}

\subsection{DigiIncubator electrical wiring}

As shown in Fig. 11, the digiIncubator electrical wiring depicts how the various electrical components are connected in the incubator. It basically illustrates how electrical power is supplied to the electrical components. This diagram gives an overview of how the power supply from the main source is distributed to the electrical components based on the power rating of each component.

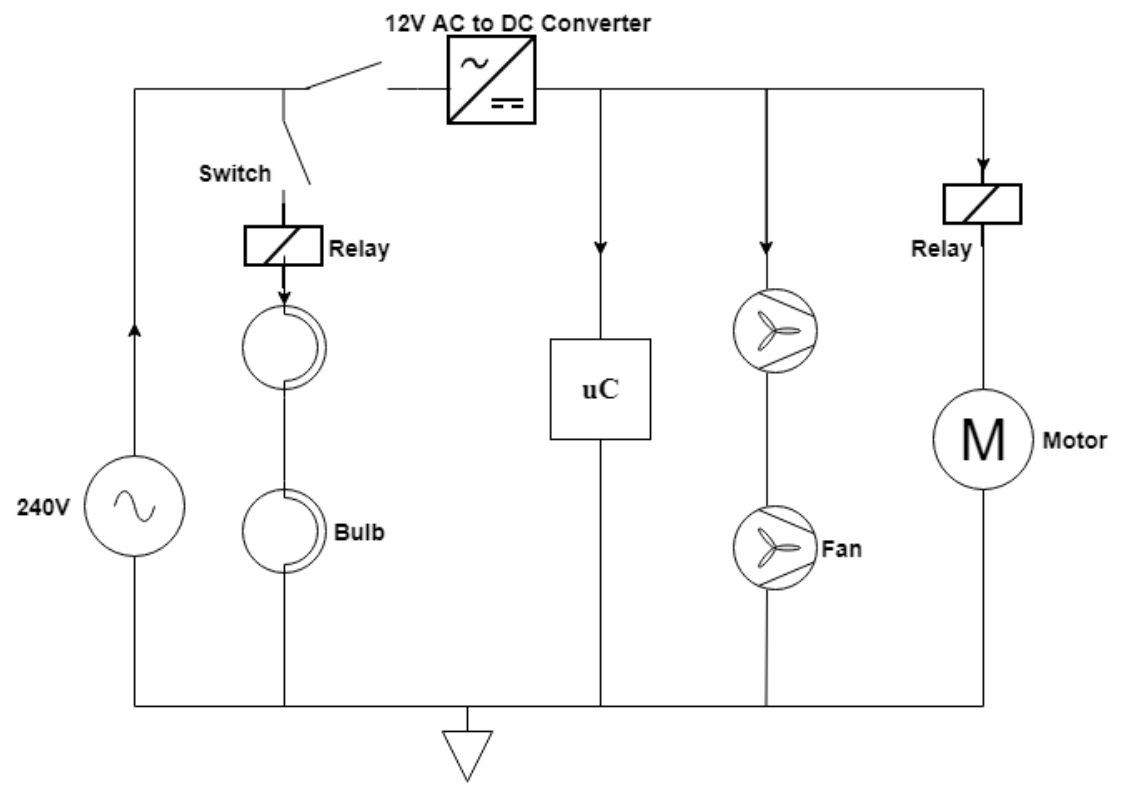

Figure 11. DigiIncubator electrical wiring

Heat energy is generated in a conductor when current flows through it. The electric current, as the result of heating effect, depends on the resistance $\mathrm{R}$ of the conductor, time $\mathrm{T}$ of current flow and the amount of current I via the conductor. A higher resistance produces more heat. The longer the time of flow, the larger the amount of heat generated. A higher amount of current produces a larger amount of heat. These three factors and their relationship can be expressed using the Joule's equation of electrical heating. This is represented by the mathematical equation in (1):

$$
H=I^{2} x R x t .
$$

The amount of heat energy generated is given by the electrical power dissipated for time $t$ as

$$
H=V x I x t=P x t
$$


From, the Joule's equation of electrical heating, it is realized that the higher the voltage and resistance rating of the heating element of choice, the more effective it is to attain the optimum temperature required for the incubation of fertile eggs.

\subsection{DigiIncubator mobile application software (digiINCU)}

The digiINCU mobile application software allows the farmer to monitor the incubation process remotely, at anywhere and at any time desired. The requirements of the mobile application were inspired by the objectives of the project. The functional requirements of the digiINCU mobile application give a detail description of what the application should do. This section presents the user requirements and other requirements others.

The functional requirements of the digiINCU mobile application software are as follows:

- Provide a login functionality to the farmer.

- Display a dashboard of successfully logged in users.

- Display any other information about all the incubators associated with the system.

- Provide QR - code for logged in users who have not yet added any incubator and others wanting to have multiple incubators.

- Provide database logging functionality for users.

The non-functional requirements of the digiINCU are:

- Performance: This refers to how fast the mobile application respond to user inputs.

- Security

- Ease of use.

Fig. 12 depicts the digiINCU Software usecase diagram. When a user successfully logged in, the user is redirected to the home screen only if he already has some registered incubator(s). Otherwise, he is redirected to the 'Add Incubator' screen which shows the QR-code scanner for the QR-code provided on the incubator.

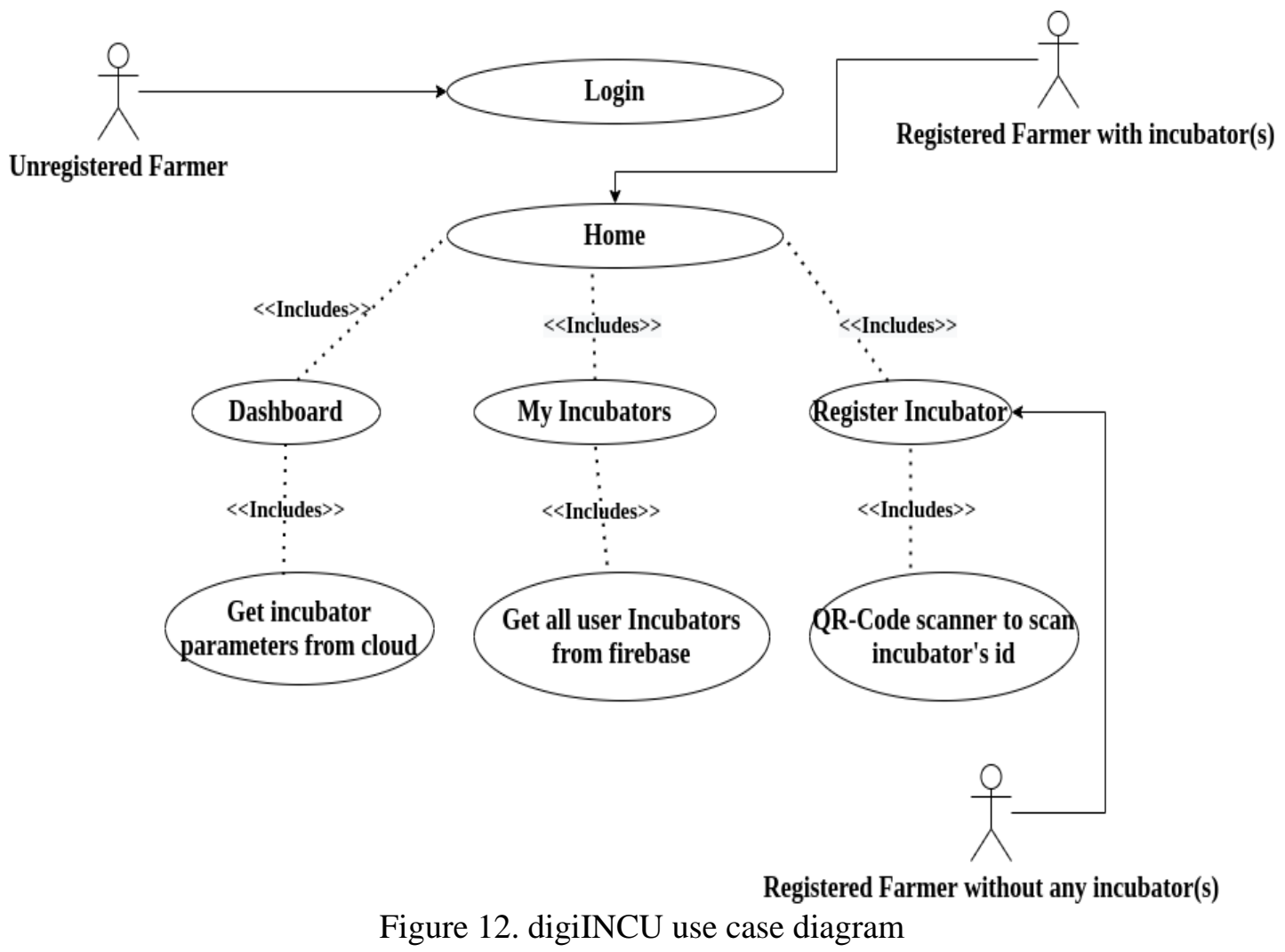

As shown in Fig. 13 at the start of the digiINCU mobile software application, the application checks for the authenticity of the user. If the check fails, the user is directed to login but if successful user is allowed access to the application. The authenticated user is redirected to the home screen if he or she has registered some incubator(s) already but if the user has not added an incubator yet, the person is redirected to a screen where he 
is presented with a QR-code scanner. He can then scan the QR-code provided on the incubator. After a successful scan of the code, he is then presented with the home screen of the application where he has access to all the details of the incubator. The user monitors the incubators from the application's dashboard and can logout any time deemed necessary.

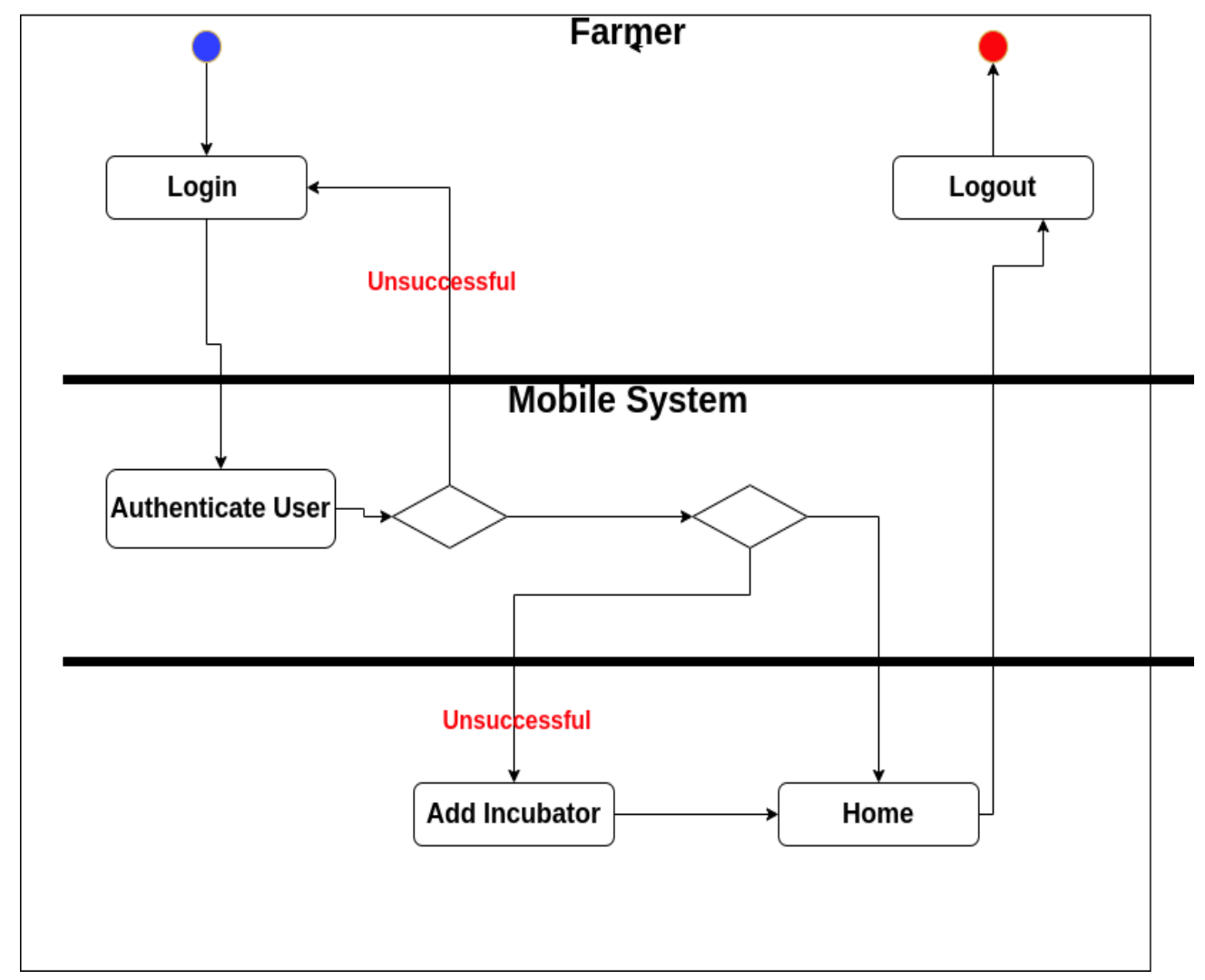

Figure 13. digiINCU activity diagram

\section{Test and evaluation}

The digiIncubator test setups are as shown in figures 14-16. The system was powered on and left for some hours. During the period of testing, the setup was analysed for accuracy in measurement of its ambient, its ability to regulate its internal environment to desired levels, the ability to turn-on on its own, the ability for it to $\log$ data to the cloud and its ability to receive commands from a user with the digiINCU mobile software application.

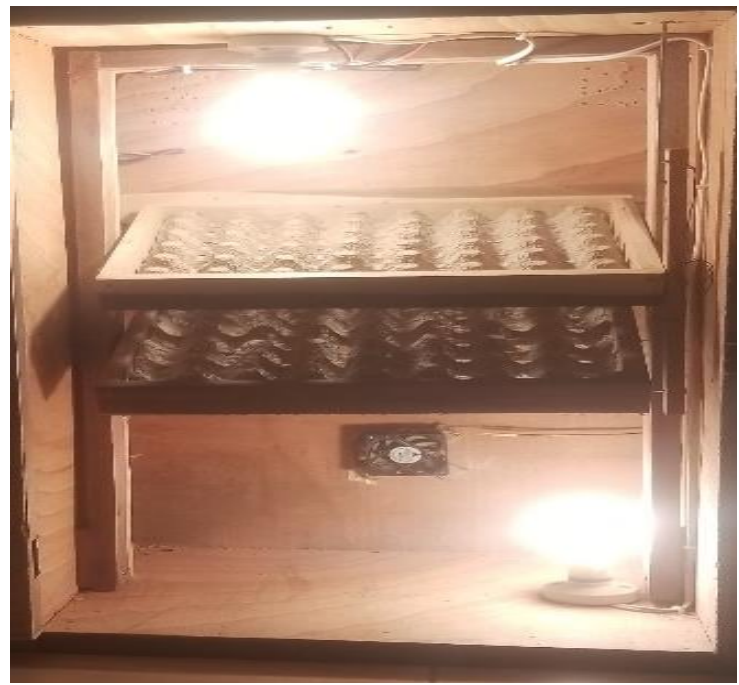

Figure 14. Opened Incubator with light on

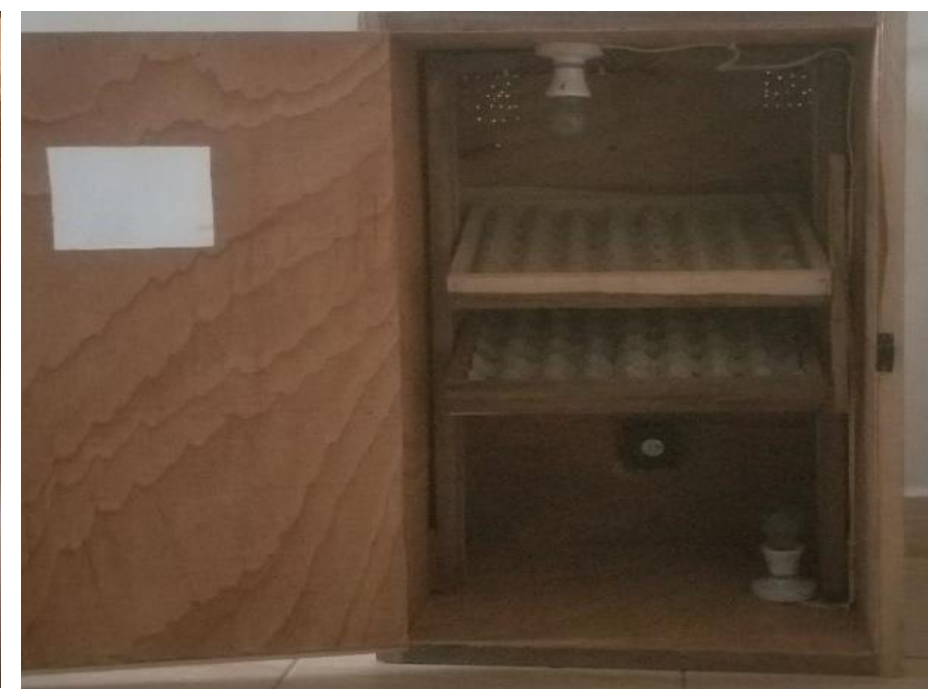

Figure 15. Opened incubator with light off 


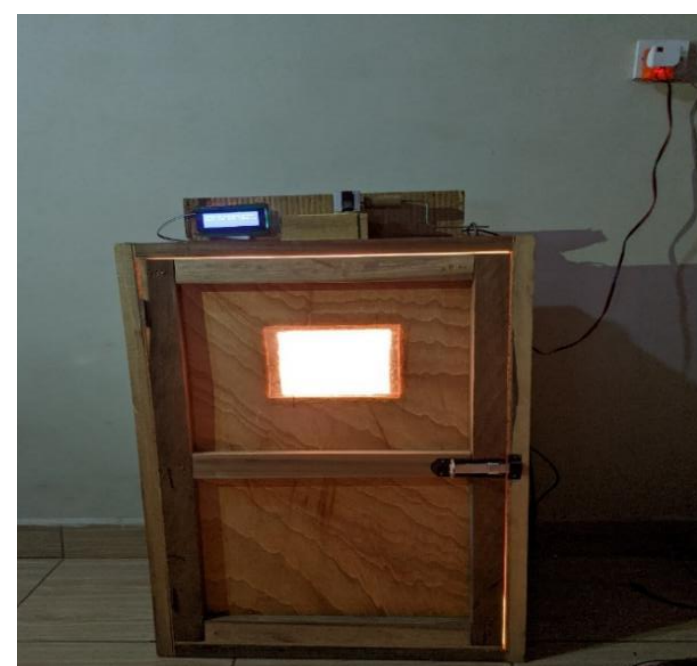

Figure 16. Built incubator incubating eggs

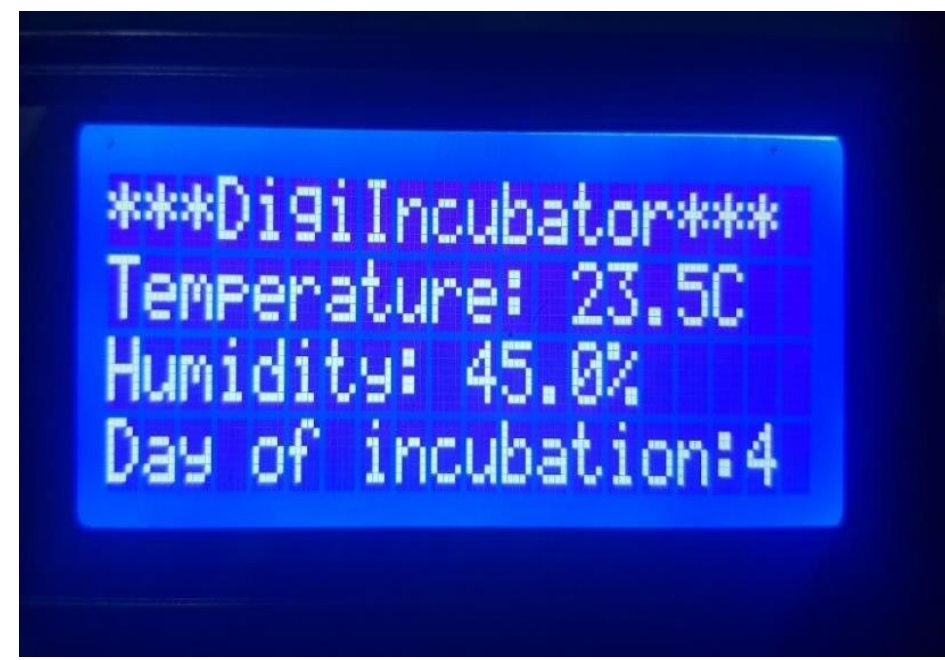

Figure 17. digiIncubator LCD displaying data

Fig. 17 shows the digiIncubator's LCD displaying data for a user present at the incubator station. It displays the temperature, humidity, and day of incubation.

Figures 18 and 19 are screen shoots of the digiINCU mobile software application used to interact with the system.

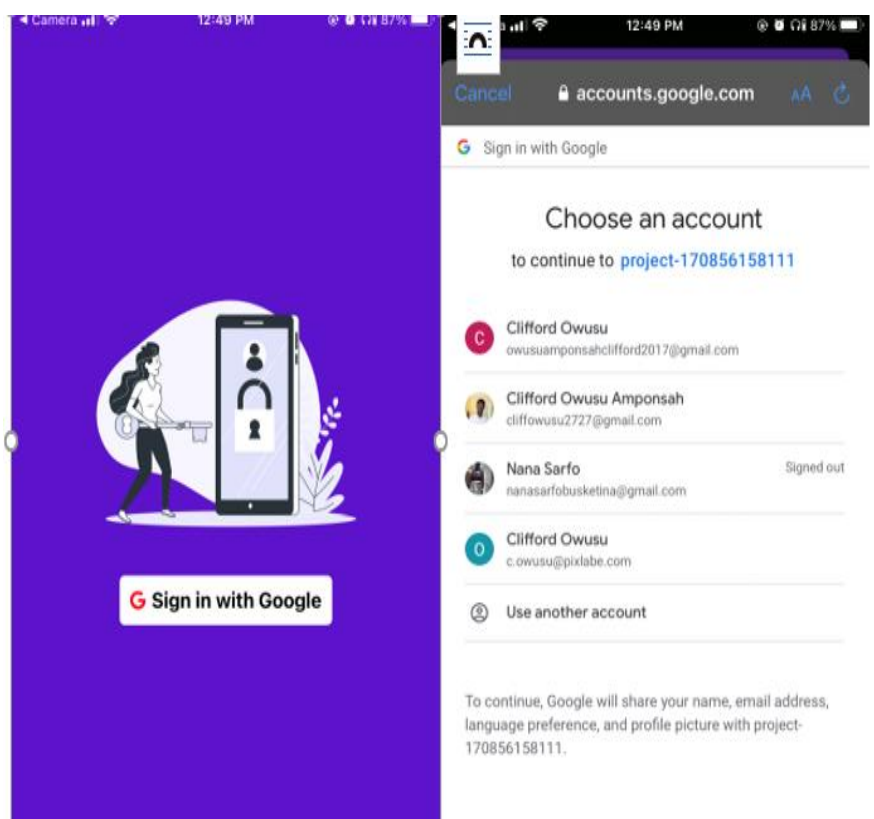

Figure18. Sign in page with Google Oauth

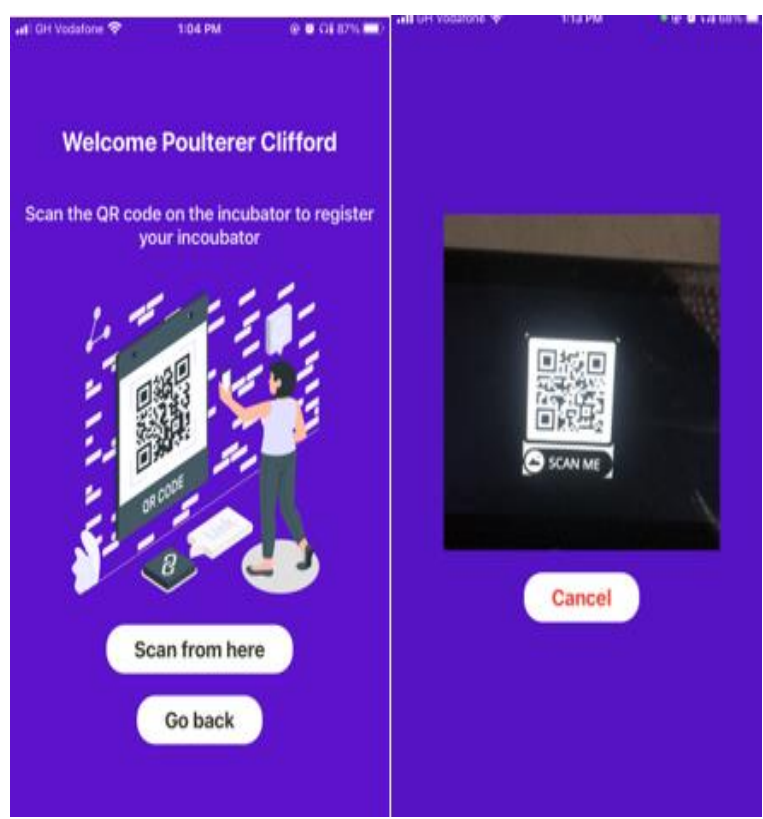

Figure 19. Add incubator screens

A single mobile application could be used to monitor multiple incubators used by a farmer as shown in Fig.20. By the operation of the system, anytime there is a change in the temperature or humidity of the incubator ambient, an HTTP post request is sent to the cloud. This request contains the recent data changes in incubator system. Data representing values of the incubator ambient are retrieved by the digiINCU mobile software application in real time. The table 2 shows sample data sent to the cloud platform via ThingSpeak during the testing period.

Table 2. DigiIncubator temperature and humidity records

\begin{tabular}{l|ll}
\hline Created at & Temperature & Humidity \\
\hline 2021-08-22 22:49:09 UTC & 25 & 16 \\
2021-08-22 22:50:44 UTC & 24 & 17 \\
2021-08-22 22:52:44 UTC & 26 & 17 \\
2021-08-22 22:54:18 UTC & 24 & 17
\end{tabular}


SEI Vol. 4, No. 1, February 2022, pp.22-33

\begin{tabular}{l|ll}
\hline Created at & Temperature & Humidity \\
\hline 2021-08-22 22:56:12 UTC & 25 & 16 \\
2021-08-22 22:57:46 UTC & 26 & 17 \\
2021-08-22 22:59:20 UTC & 25 & 16 \\
2021-08-22 23:00:54 UTC & 27 & 16 \\
$2021-08-22$ 23:02:29 UTC & 25 & 16 \\
2021-08-22 23:04:03 UTC & 24 & 17 \\
$2021-08-22$ 23:06:22 UTC & 25 & 16 \\
2021-08-22 23:07:57 UTC & 24 & 17 \\
\hline
\end{tabular}

The ambient conditions recorded by the system is shown in a form of graphs in Fig. 21.

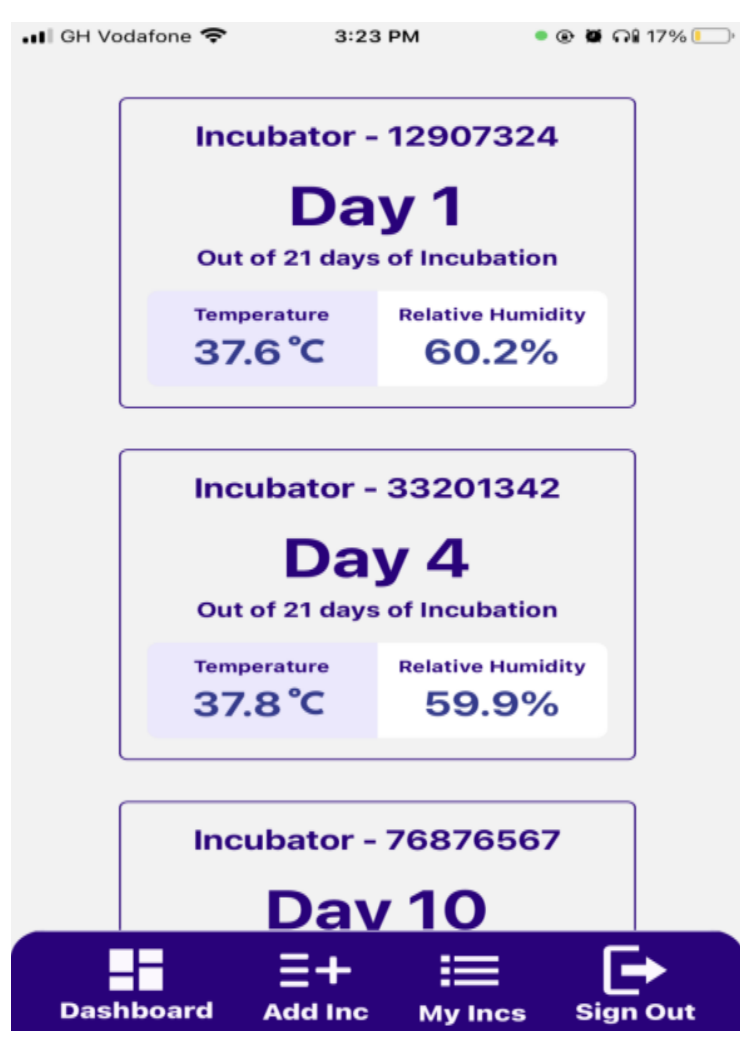

Figure 20. DigiIncubator screen

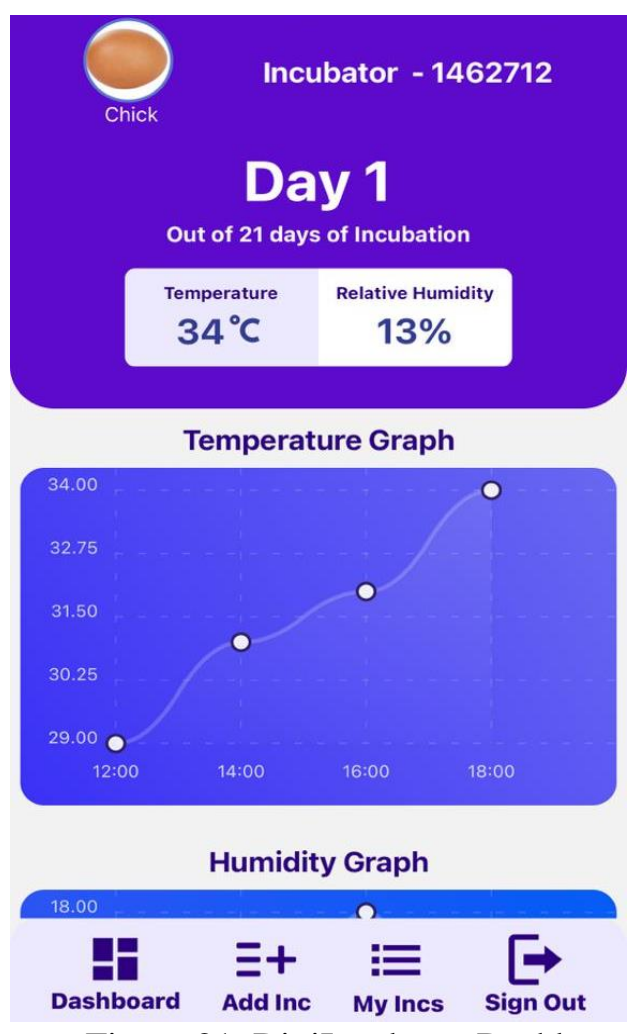

Figure 21. DigiIncubator Dashboard

DigiIncubator system data collected are sent from the microcontroller to the cloud server. With the aid of ThingSpeak, collected data are displayed in form of graphs. Fig. 22 depicts temperature against time and Fig. 23 shows humidity against time.

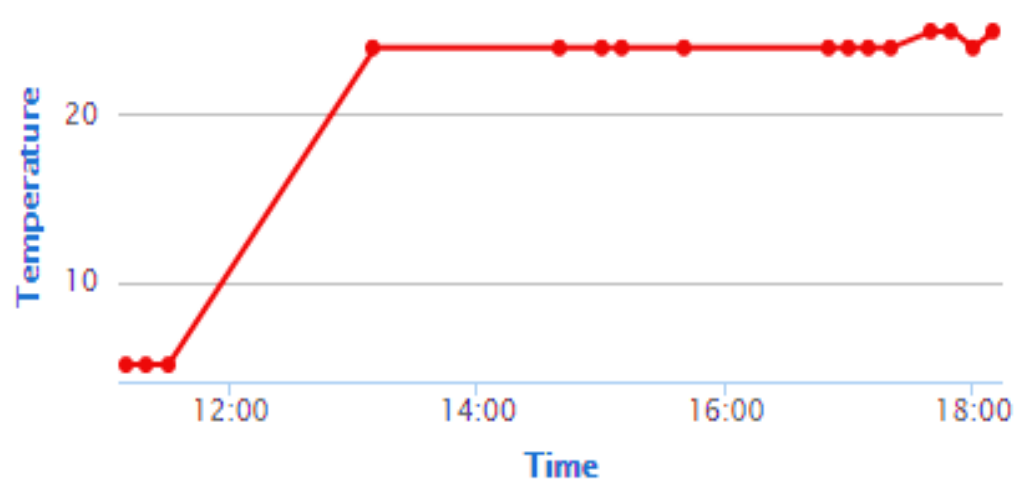

ThingSpeak.com

Figure 22. DigiIncubator: ThingSpeak temperature vrs. time 


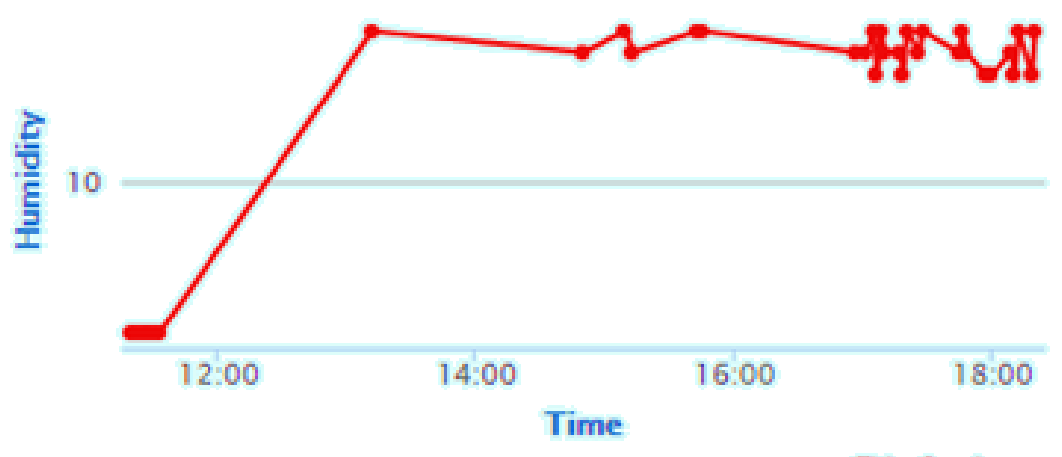

Figure 23. DigiIncubator: ThingSpeak humidity vrs. time

\section{Conclusion}

This project was initiated to solve the issue of expensive egg incubators in Ghana. It was observed that the expensive incubator problem prevented many farmers from patronizing incubators to increase the number of eggs that are hatched in their farms. This paper researched, implemented, and tested an efficient incubator that is less expensive and smart. The system proved smart by being able to monitor, report and automatically regulate its internal environment within the required limits without the farmer's presence and periodically turn eggs inside of it as such, ensuring that the eggs were well incubated thus producing high hatchability. In all this, the price of the incubator remained low as compared with incubators in the market. This work contributes to the Sustainable Development Goal (SDG) Number 2'. Future works on this would investigate adding an ecofriendly and cheap power source such as solar power to the system so that farmers in areas with unstable power supply can use it.

\section{Declaration of competing interest}

The authors declare that they have no any known financial or non-financial competing interests in any material discussed in this paper.

\section{References}

[1] B. Tanko, Revamping the Poultry Sector in Ghana, Ministry of Food and Agriculture, Oct. 2019. Accessed on Dec. 4,2020. [Online]. Available: https://www.mofa.gov.gh/site/mediacentre/agricultural-articles/321-revamping-the-poultry-sector-in-ghana

[2] L. Y. Kusi, S. Agbeblewu and A. I. Kwadwo, K. Minta Nyarku, "The Challenges and Prospects of the Commercial Poultry Industry in Ghana: A Synthesis of Literature", International Journal of Management Sciences, vol. 5, no.6, pp. 476-489, 2015.

[3] N. Berkhout, Millions invested to revive Ghana's poultry sector, Poultry World, May 2020. Accessed on Dec. 14, 2020. [Online]. Available: https://www.poultryworld.net/Meat/Articles/2020/5/Millionsinvested-to-revive-Ghanas-poultry-sector-588246E/

[4] “Incubator Terminology Explained," Mar. 2017. Accessed on Dec. 14,2020. [Online]. Available: https://www.surehatch.co.za/pages/incubator-terminology-explained

[5] "Science of Incubation," Apr. 2016. Accessed on: Dec. 14,2020. [Online]. Available: https://www.sites.ext.vt.edu/virtualfarm/poultry/poultry_incubation_science.html\#: :text= Incubation $\% 20$ means $\% 20$ maintaining $\% 20$ conditions $\% 20$ favorable, humidity $\% 2 \mathrm{C} \% 20$ ventilation $\% 2 \mathrm{C}$ $\% 20$ and\%20turning

[6] H. S. Wilgus and W. W. Sadler "Incubation Factors Affecting Hatchability of Poultry Eggs," Poultry Science, vol. 33, no.3, pp. 460-471, 1954.

[7] Harb, S. K. Habbib, Y.A. Kassem, A.M. and A. Raies (2010). "Energy Consumption for Poultry Egg Incubator to Suit Small Farmer,” Egypt Journal Agricultural Research, vol. 88, no.1, pp. 193-210, 2010.

[8] E. Corti and E. Vogelaar, The Oldest Hatcheries are Still in Use, Aviculure-Europe, Aug. 2012. Accessed on: Jan. 01, 2021. [Online]. Available: http://www.avicultureeurope.nl/nummers/12e03a08.pdf 
[9] S. Shittue, A. S. Muhammad and O. Jimoh. "Development of an Automatic Bird-Egg Incubator," A Journal of Embedded System \& Applications. vol. 5, no.1, pp:1-10, 2017.

[10] D. Nedelkovski, DHT22 Sensors Temperature and Humidity Tutorial using Arduino, How to Mechatronics, Mar. 2016. Accessed: Dec. 20,2020. [Online]. Available: https://howtomechatronics.com/tutorials/arduino/dht11-dht22-sensors-temperature-and-humiditytutorial-using-arduino/

[11] L. K. S. Tolentino, E. Justine G. Enrico, R. L. M. Listanco, M. Anthony M. Ramirez, T. L. U. Renon and M. Rikko B. Samson, "Development of Fertile Egg Detection and Incubation System Using Image Processing and Automatic Candling, "TENCON 2018 - 2018 IEEE Region 10 Conference, 2018, pp. 0701-0706, doi: 10.1109/TENCON. 2018.

[12] H. Sunitha, L. Niranjan, D. P. B. Rajesh, R. Pooja and B. K. Supritha, "Universal Egg Incubation System for Hatching using Atemga328P, Proteus Design Tool and IoT", International Journal of Research and Analytical Reviews (IJRAR), vol. 7, P- ISSN 2349-5138. 2020.

[13] P. E. Okpagu and A. W. Nwosu," Development and Temperature Control of Smart Egg Incubator System for Various Types of Egg”, European Journal of Engineering and Technology, vol. 4, no.2, ISSN 2056-5860, 2016.

[14] I. O. Olaoye, B. M. Lawal, S.O Ibrahim and B. A. Sanusi, "An Electrically Operated Incubator for Household". Greener Journal of Science and Technological Research, vol. 3, no 5, pp. 160-165, 2013.

[15] A. B. Umar, K. Lawal and M. Mukhtar and M. S. Adamu, "Construction of an Electrically Operated Egg Incubator". International Journal of Modern Engineering Sciences, vol.5, no.1, pp 1-18, 2016.

[16] A. A. Sunday, O. A. Ogunbode, E. G. Babatunde, A. M. Olalekan, "Design and Construction of Automated Eggs Incubator for Small Scale Poultry Farmers" International Journal of Technical Research \& Science, vol. 5, ISSN no.:2454-2024, 2020.

[17] H. D. Fordjour, J. A Hamidu, K. Adomako, “Assessing Incubation and Performance Deficiencies to Boast Broiler Production.” American Research Journal of Agriculture, vol. 3, pp:1-6, 2017.

[18] S. Kassandra, "Everything You Need to Know About Fertile Eggs", Backyard Chicken Coops, Sep. 2020, Accessed: Dec. 13,2020. [Online]. Available: https://www.backyardchickencoops.com.au/blogs/learning-centre/everything-you-need-to-knowabout-fertile-egg

[19] "Artificial Incubation," Apr. 15,2009. Accessed: Dec. 14,2020. [Online]. Available: https://www.thepoultrysite.com/articles/artificial-incubation

[20] G. T. Pereira, E. S. Nakage, J. P Cardozo, "Effect of Temperature on Incubation Period, Embryonic Mortality, Hatch Rate, Egg Water Loss and Partridge Chick Weight (Rhynchotus rufescens)", Brazilian Journal of Poultry Science, v. 5, no.2, pp: 131-135, 2003.

[21] S. G. Oliveira, M. V. D. Santos (2020), "Effects of different egg turning frequencies on incubation efficiency parameters" Poultry Science, vol. 99, pp. 4417-4420, 2020. 